\title{
lesson of the month (2)
}

\section{A curious case of ST elevation}

Takotsubu cardiomyopathy (TC) describes transient left ventricular apical ballooning due to akinetic myocardium. It mimics acute coronary syndrome, with similar electrocardiogram changes and raised troponin, but visible coronary artery stenosis is missing. Patients usually recall a preceding stressful event and post-menopausal women are at greatest risk. Aetiology of TC remains contentious, but the strongest theory suggests increased sympathetic nervous system activity resulting in elevated catecholamines, which culminates in cardiac manifestations. Treatment is supportive and complications include arrhythmias, cardiac rupture, thrombus formation and congestive cardiac failure. The prognosis is favourable and by definition cardiac function returns to pre-morbid levels. Mortality is rare $(1-2 \%)$.

\section{Case presentation}

A 69-year-old female presented to the emergency department with acute left sided chest pain and dyspnoea, which started after an argument. She had no significant medical history or regular medication. Electrocardiogram (ECG) showed right bundle branch block and ST elevation in the anterolateral leads. A diagnosis of ST elevation myocardial infarction was made. She received appropriate treatment and was transferred for primary angioplasty. Subsequent left ventriculogram showed apical ballooning and hyper-contractile basal segments (Fig 2).

Based on these findings, a diagnosis of takotsubu cardiomyopathy (TC) was made. Biochemical investigations showed an elevated troponin, while cardiac magnetic resonance imaging (MRI) demonstrated akinetic apical segments and hyper-contractile basal segments. Overall left ventricle (LV) systolic function was severely impaired. However, late gadolinium imaging showed no late enhancement; proving viable cardiomyocytes.

The patient was commenced on standard heart failure therapy and discharged home after an uncomplicated 5-day admission. At follow up she remained well and a repeat cardiac MRI scan after 2 months showed complete LV recovery.

Gary Sharp, ${ }^{1}$ foundation year two doctor; Roshan Weerakody, ${ }^{2}$ consultant cardiologist; Williams Ricketts, ${ }^{3}$ clinical teaching fellow; Zane Perkins, ${ }^{4}$ research fellow

${ }^{1}$ North-east Thames London deanery, Broomfield Hospital, Chelmsford, Essex, UK; ' ${ }^{2}$ London Chest Hospital, London, UK; ${ }^{3}$ Newham University Hospital, Bart's Health NHS Trust, Plaistow, London, UK; ${ }^{4}$ Centre for Trauma Sciences, Queen Mary University London, UK

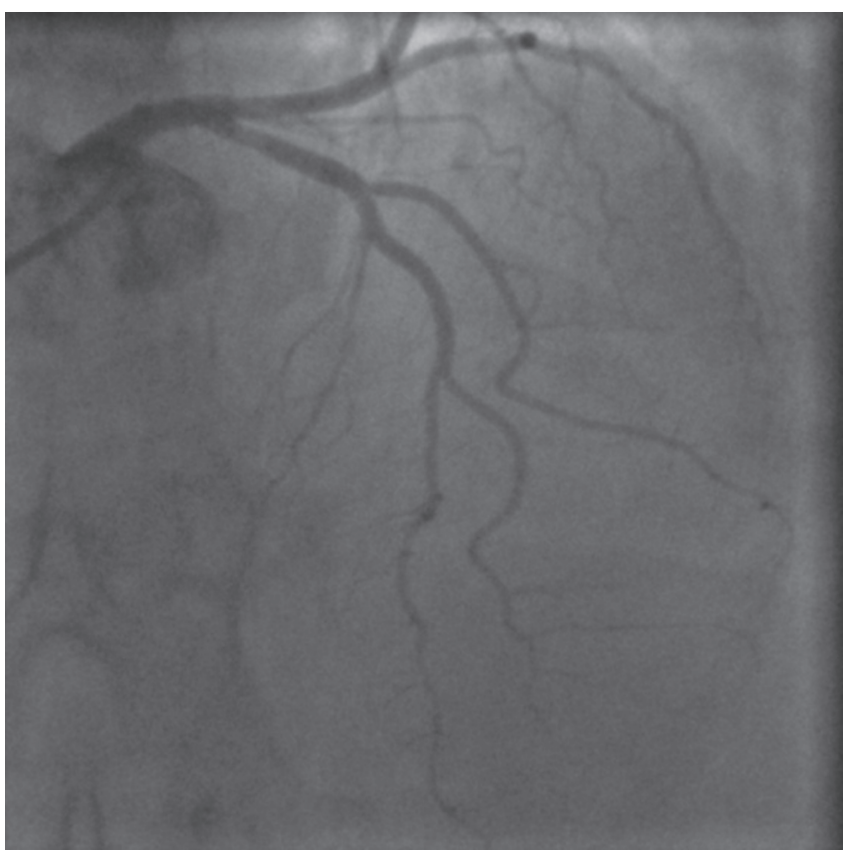

Fig 1. Coronary angiography showed no significant culprit lesion.

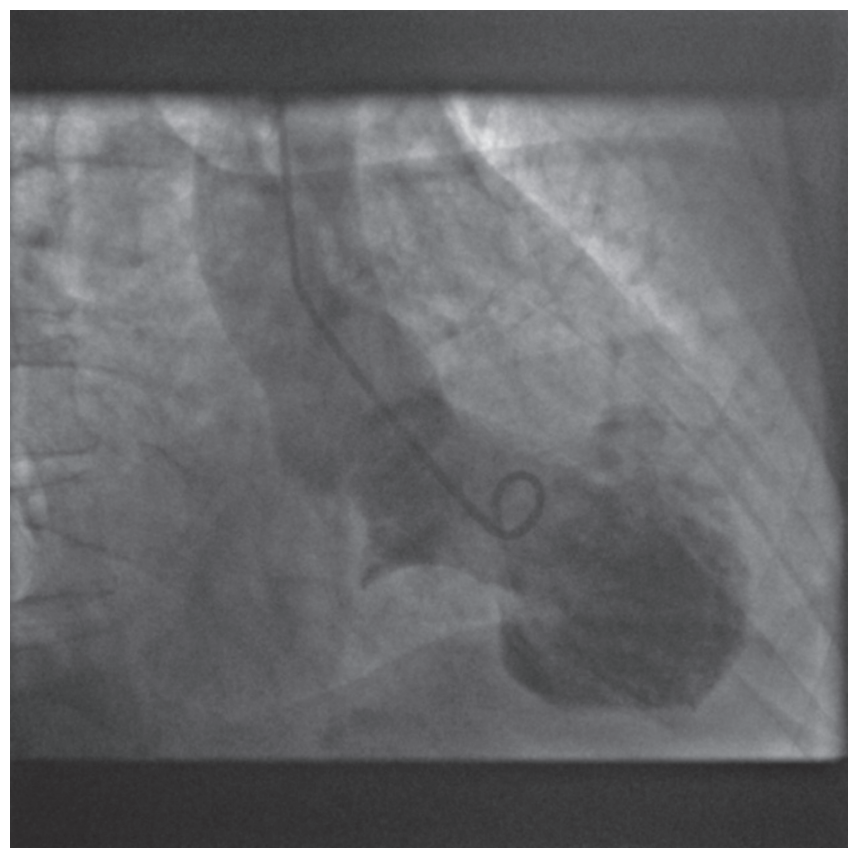

Fig 2. Left ventriculogram. 


\section{Lesson}

Takotsubu cardiomyopathy was first described in $1991^{1}$ and termed takotsubo by the Japanese in reference to the fishing pots used to catch octopi, which resemble the left ventricular apical ballooning due to akinetic myocardium and the hypercontractile cardiac base seen on ventriculogram during systole. ${ }^{2,3}$ TC has several pseudonyms including 'stress cardiomyopathy', and 'broken heart syndrome'.

The presentation of TC mimics acute coronary syndrome (ACS), ${ }^{2}$ but without significant coronary artery stenosis seen on angiogram. ${ }^{2}$ The majority of patients are post-menopausal women, and men account for as little as $10 \%$ of cases. ${ }^{2}$ The true prevalence is unknown, but is estimated at $1-2 \%$, and may account for up to $2 \%$ of all ACS presentations. ${ }^{2}$ Individuals may describe recent intense emotional or physical stress prior to the onset of symptoms and the majority of patients report a physical stressor as the precipitating event. However, up to one-third of TC patients are unable to identify any preceding stressful event. $^{2}$

\section{Clinical presentation}

Diagnostic criteria for $\mathrm{TC}^{4}$ are shown in Box 1. All criteria must be met to diagnose TC. The most common presenting symptom is cardiac chest pain (70-90\%) followed by dyspnoea (20\%). Severe cases may be complicated by pulmonary oedema, arrhythmias and cardiogenic shock. ${ }^{5}$

Electrocardiogram abnormalities are transient, with anterior lead ST elevation most frequently encountered. ${ }^{5}$ ST elevation prompts rapid angiography and thus coronary patency is visualised leading to ACS exclusion. ${ }^{5}$ Deep $\mathrm{T}$ wave inversion is commonly seen a few days following initial presentation. ${ }^{3}$

Patients diagnosed with TC present with mildly raised troponin I levels, ${ }^{2,3}$ which peak at around $24 \mathrm{~h}$ post chest pain onset. ${ }^{4,5}$ Levels are not as elevated as those found in ACS and normalise quickly, unlike myocardial infarction. ${ }^{4-6}$

Left ventricular wall ballooning predominantly affects the apex $(82 \%)$ and mid-ventricular myocardium, with relative sparing of the base. ${ }^{2}$ There are exceptions and patients may have apical sparing, or indeed biventricular akinetic dysfunction, the latter having a poorer prognosis. ${ }^{2,3}$ Cardiac function and left ventricular wall motion typically return to normal within days to weeks. ${ }^{4}$

\section{Pathophysiology}

Takotsubu cardiomyopathy is idiopathic, ${ }^{2,5}$ but the most favoured hypothesis is over-activation of the sympathetic nervous system and subsequently increased catecholamine release, especially adrenaline. ${ }^{4}$ Plasma catecholamine concentration is elevated in TC when compared to ACS. But not all TC patients describe a preceding stressful event, thus levels of catecholamines may not have been elevated. ${ }^{2}$
Box 1. Modified Mayo Clinic diagnostic criteria. (Reproduced with permission of Prasad et al, 2008). ${ }^{4}$

1 Transient hypokinesis, akinesis or dyskinesis of the left ventricular mid segments, with or without apical involvement. Regional wall motion abnormalities extend beyond a single coronary bed.* A preceding physical or emotional stressor is often present.

2 No obstructive coronary disease or acute plaque rupture (determined angiographically). ${ }^{\dagger}$

3 New ECG changes (ST segment elevation, T wave inversion or both) or modest elevation in cardiac troponin level.

4 No phaeochromocytoma or myocarditis. For such patients, the diagnosis of TC should be made cautiously and a clear, stressful, precipitating event must be sought.

ECG = electrocardiogram; TC = takotsubu cardiomyopathy.

*A rare exception to these criteria is regional wall motion abnormality that is

limited to one coronary territory.

†A patient with obstructive coronary atherosclerosis may also have TC

development. However, this is very rare.

Several theories exist regarding TC aetiology, including:

1 Raised catecholamines cause epicardial coronary vasospasm. ${ }^{4}$

2 Catecholamines affect endothelial cell function reducing myocardial blood supply. ${ }^{3}$

3 Transient ischaemia secondary to a transient thrombosis in an extended left anterior descending coronary artery (LAD) occurs. The extended LAD hypothesis states that because the ventricular wall motion abnormalities seen in TC do not correspond to a single coronary artery territory, an extended $\mathrm{LAD}$ is present, which correlates to the area of akinetic left ventricle. ${ }^{7}$

However, these hypothetical aetiologies are either rarely seen, ${ }^{3}$ discredited via angiography ${ }^{5}$ or not well supported in current literature. $^{2}$ No genetic link has been found. ${ }^{5}$

\section{Complications and treatment}

The mainstay of management involves supportive treatment. Haemodynamically stable patients are treated with angiotensin converting enzyme inhibitors (ACEi), beta-blockers and diuretics. ${ }^{4}$ Haemodynamically unstable patients may require intraaortic balloon pumps. ${ }^{3}$

Left ventricular outflow tract obstruction may arise due to the abnormal hyperkinetic myocardial base and pressure changes between the left ventricle and the adjacent left atrium. These pressure changes cause medial deviation of the mitral valve towards the ventricular septum. ${ }^{7}$ Outflow tract obstruction ensues, manifesting as profound hypotension. ${ }^{4}$ Beta-blockers may reduce the contractility of myocytes within the cardiac base, alleviating some of the outflow obstruction. ${ }^{4,7}$

Judicious use of intravenous fluid is recommended as this may increase blood pressure and thus stroke volume. Likewise, it may exacerbate congestive cardiac failure (CCF). ${ }^{4} \mathrm{CCF}$ is the most common complication and is more common in those presenting with right ventricular akinesis/hypokinesis (20\%) 
and may manifest as pulmonary oedema $(22 \%),{ }^{5}$ warranting diuretics. ${ }^{4}$

Left ventricle thrombi may form in the akinetic ventricle. ${ }^{8}$ Prophylactic anticoagulation in those with apical hypo-/akinesis is indicated. ${ }^{3}$ Cardiac rupture is rare and most commonly affects the left ventricular wall. Beta-blockers may reduce intraventricular pressure and load, thus reducing possible rupture. ${ }^{9}$ Several arrhythmias have been described but these are uncommon. ${ }^{4}$

\section{Prognosis}

Prognosis of TC is favourable, with a low rate of recurrence (1-11\%). ${ }^{3}$ One study showed that 4 -year survival was no different to an age- and sex-matched population. ${ }^{3}$ However, as this is a relatively new condition, sufficient long-term data is not available. Ejection fraction and cardiac function, by definition, return to pre-morbid levels, ${ }^{2,4}$ as long as no other simultaneous cardiac insult has occurred. Cardiology follow up is advised to ensure complete recovery. ${ }^{4}$ Mortality is rare $(1-2 \%)^{4,5}$ and mostly due to ventricular fibrillation ${ }^{2}$ or cardiac rupture. ${ }^{9}$

\section{Conclusion}

Heightened awareness of TC will improve diagnostic sensitivity and should also lead to more appropriate treatment. TC is not always preceded by a notable stressful event. Biventricular involvement should alert the clinician to potentially greater morbidity.

\section{References}

1 Dote K, Sato H, Tateishi H et al. Myocardial stunning due to simultaneous multivessel coronary spasms: A review of 5 cases. J Cardiol 1991;21:203-214.

2 Eitel I, Knobelsdorff-Brenkenhoff F, Bernhardt P et al. Clinical characteristics and cardiovascular magnetic resonance findings in stress (takotsubo) cardiomyopathy. JAMA 2011;306;277-286.

3 Wittstein I. Acute stress cardiomyopathy. Curr Heart Fail Rep 2008;5:61-68.

4 Prasad A, Lerman A, Rihal CS. Apical ballooning syndrome (TakoTsubo or stress cardiomyopathy): a mimic of acute myocardial infarction. Am Heart J 2008;155:408-417.

5 Hurst T, Prasad A, Askew JW 3rd et al. Takotsubo cardiomyopathy: a unique cardiomyopathy with variable ventricular morphology. JACC Cardiovasc Imaging 2010;3:641-649.

6 Sealove B, Tiyyagura S, Fuster V. Takotsubo cardiomyopathy. J Gen Intern Med 2008;23:1904-1908.

7 Derrick D. The 'broken heart syndrome': understanding takotsubo cardiomyopathy. Crit Care Nurse 2009;29:49-58.

8 de Gregorio C, Grimaldi P, Lentini C. Left ventricular thrombus formation and cardioembolic complications in patients with takotsubolike syndrome: a systematic review. Int J Cardiol 2008;131:18-24

9 Kumar S, Kaushik S, Nautiyal A et al. Cardiac rupture in takotsubo cardiomyopathy: a systematic review. Clin Cardiol 2011;34:672-676.

Address for correspondence: Dr G Sharp, Court Road,

Broomfield, Chelmsford, Essex CM1 7ET.

Email: garybeau1@yahoo.co.uk

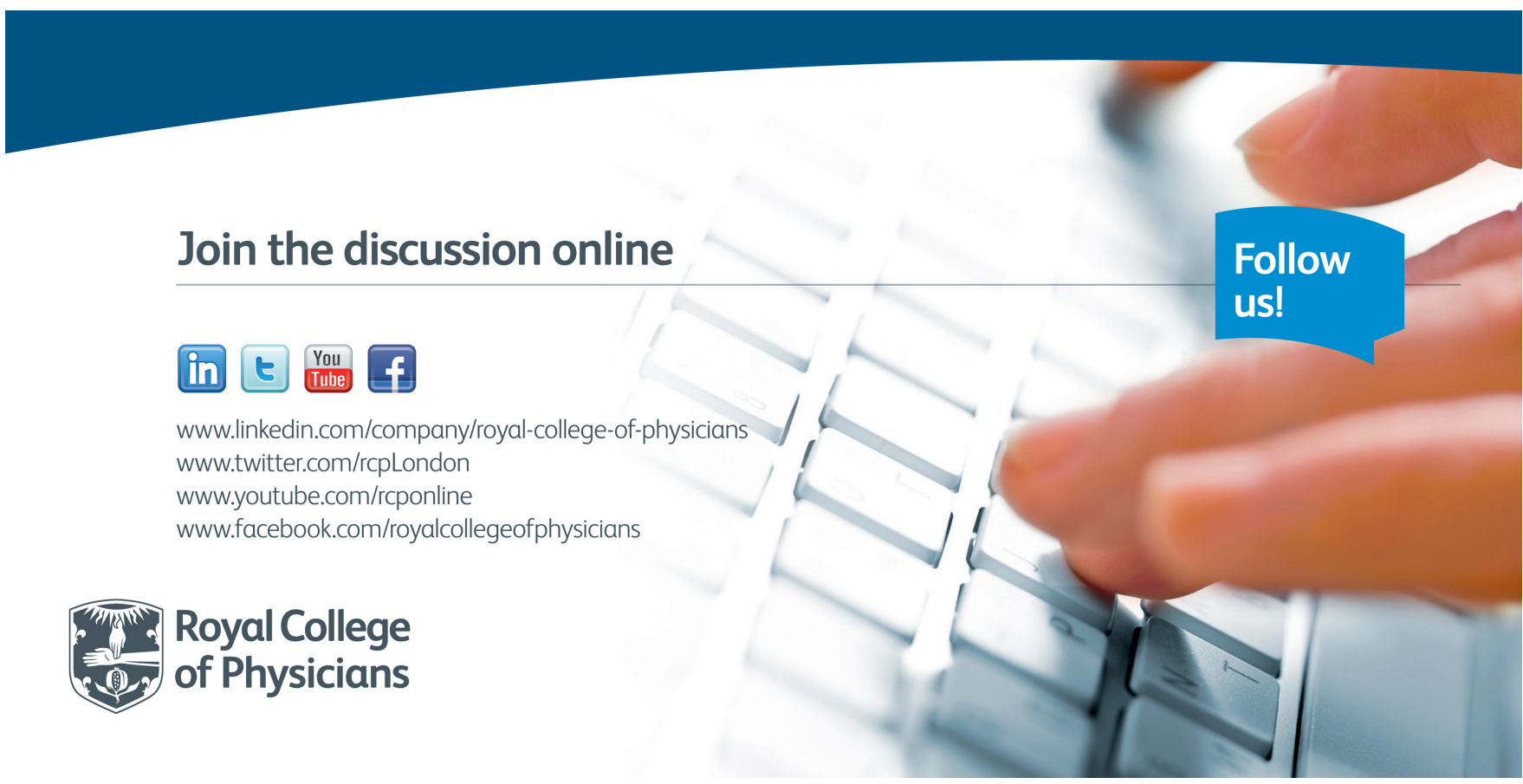

